\title{
STABILITY OF SUPERPOSED FLUIDS THROUGH MAGNETIC FIELD WITH SUSPENDED PARTICLES OF DIFFERENT PERMEABILITY SATURATED THROUGH POROUS LAYER
}

\author{
M. SINGH \\ Department of Mathematics \\ Govt. Post Graduate College Seema (Rohru) \\ Distt Shimla (H.P) -171207, INDIA \\ E-mails: mahinder_singh91@rediffmail.in \\ drmsmath78@gmail.com
}

\begin{abstract}
The instability of plane interface between two superposed Rivlin-Ericksen elastico-viscous fluids saturated through a porous medium has been studied to include the suspended (dust) particles effect. Following the linearized stability theory and normal mode analysis the dispersion relation is obtained. For stationary convection, the Rivlin-Ericksen elastico-viscous fluid behaves like Newtonian fluids. It found that for a potentially stable arrangement the Rivlin-Ericksen elastico-viscous fluid of different permeabilities in the presence of suspended particles in a porous medium is stable, whereas in a potentially unstable case instability of the system occurs. In the presence of a magnetic field for a potentially stable arrangement the system is always stable and for the potentially unstable arrangement, the magnetic field succeeds in stabilizing certain wave-number band which was unstable in the absence of the magnetic field.
\end{abstract}

Key words: porous medium, suspended particles, Rivlin-Ericksen fluid and magnetic field.

\section{Introduction}

The influence of the viscosity on the stability of a plane interface separating two incompressible superposed conducting fluids of uniform densities, when the whole system is acted on by a uniform magnetic field, was studied by Bhatia (1974). Chandra (1938) observed a contradiction between the theory for the onset of convection in fluids heated from below and his experiments. He performed the experiment in an air layer and found that the instability depended on the depth of the layer. A Bénard-type cellular convection with fluid descending at the cell centre was observed when the predicted gradients were imposed, for layers deeper than $10 \mathrm{~mm}$. A convection which was different in character from that in deeper layers occured at much lower temperature gradient than predicted, if the layer depth was less than $7 \mathrm{~mm}$ and was called columnar instability. He added an aerosol to mark the flow pattern. Scanlon and Segel (1973) studied the effect of suspended particles on the onset of Bénard convection and found that the critical Rayleigh number was reduced solely because the heat capacity of the pure gas was supplemented by that of the particles. Sharma et al. (1976) studied the effect of suspended particles on the onset of Bénard convection in hydromagnetics. The effect of suspended particles was found to destabilize the layer whereas the effect of the magnetic field was stabilizing. Generally, the magnetic field has a stabilizing effect on the instability but there are a few exceptions. For example, Kent (1966) studied the effect of a horizontal magnetic field, which varies in the vertical direction, on the stability of parallel flows and showed that the system is unstable under certain conditions, while in the absence of the magnetic field the system is known to be stable.

With a growing importance of the effect of suspended particles on the stability of superposed fluids in the field of industrial and chemical engineering, Sharma (1976) studied the thermal instability of a layer of Oldroydian viscoelastic fluid acted on by a uniform rotation whereas Sharma and Sharma (1977) studied the 
instability of the plane interface separating two Oldroydian viscoelastic superposed fluids of uniform densities.

In all the above studies, the fluids have been considered to be Newtonian or viscoelastic (Maxwellian or Oldroydian). There are many elastico-viscous fluids that cannot be characterized by Maxwell's or Oldroyd's constitutive relations. One such fluid is the Rivlin-Ericksen elastico-viscous fluid (Rivlin and Ericksen, 1955). The studies of the flow of non-Newtonian fluids having uniform distribution of the dust particles such as latex particles in emulsion paints, reinforcing particles in polymer melts and rock crystals in molten lava are quite useful. Srivastava (1971) analyzed the unsteady flow of the Rivlin Ericksen fluid with uniform distribution of dust particles through a circular cylinder under the influence of a time varying pressure gradient. Srivastava and Singh (1988) studied the unsteady flow of a dusty elastico-viscous Rivlin -Ericksen fluid through a channel of different cross-sections in the presence of a time-dependent pressure gradient. Kumar et al. (2007), studied hyderodynamic and hyderomagnetic stability of the Rivlin-Ericksen fluid and found that growth rates are found to decrease as well as increase with the increase in kinematic viscosity and kinematic visco-elasticity without a magnetic field and with a magnetic field, for the stable-stratification, the system is found to be stable or unstable under certain conditions. Singh and Gupta (2011) studied thermal instability of the Rivlin-Ericksen fluid permeated with suspended particles in hyderodynamics in a porous medium and found that the magnetic field has only a stabilizing effect, whereas medium permeability has a destabilizing effect for the case of stationary convection. Gupta et al. (2012) studied thermal convection of the Rivlin-Ericksen fluid with hall currents and found that the magnetic field is found to have a stabilizing effect on the system.

Keeping in mind the importance of non-Newtonian fluids in geophysical fluid dynamics, chemical technology and petroleum industry, the hydrodynamic and hydromagnetic stability of superposed RivlinEricksen elastico-viscous fluids of different permeability with suspended particles in a porous medium is considered. In the interior of the Earth, the magnetic field may be variable and may altogether alter the nature of the instability. The Coriolis force also plays an important role in the stability of geophysical phenomena owing to the importance of a variable magnetic field force us to study this problem.

\section{Formulation of the problem}

Let $\rho, p$ and $\boldsymbol{q}(u, v, w)$ denote respectively the density, pressure and filter velocity of the pure fluid; $\boldsymbol{q}_{d}(x, t)$ and $N(x, t)$ denote the velocity and number density of the suspended particles, $\boldsymbol{q}_{d}=(l, r, s), \bar{x}=(x$, $y, z)$ and $\lambda=(0,0,1)$, respectively. $K=6 \pi \rho v \eta$, where $\eta$ being the particle radius, is Stokes's drag coefficient. Let $\in, k_{1}, \mu, \mu^{\prime}$ and $g$ stand for the medium porosity, medium permeability, viscosity of the fluid, viscoelasticity of the fluid and acceleration due to gravity, respectively. Then the equations of motion and continuity for the Rivlin Ericksen viscoelastic fluid permeated with suspended particles through a porous medium, Scanlon and Segal (1973), Sharma and Kumar (Sharma and Kumar, 1997) are

$$
\begin{aligned}
& \frac{\rho}{\epsilon}\left[\frac{\partial \boldsymbol{q}}{\partial t}+\frac{1}{\epsilon}(\boldsymbol{q} \cdot \nabla) \boldsymbol{q}\right]=-\nabla p-\rho g \lambda+\frac{K N}{\epsilon}\left(\boldsymbol{q}_{d}-\boldsymbol{q}\right)-\frac{1}{k_{l}}\left(\mu+\mu^{\prime} \frac{\partial}{\partial t}\right) \boldsymbol{q}, \\
& \nabla \cdot \boldsymbol{q}=0 .
\end{aligned}
$$

In Eq.(2.1), an assumption has been made of uniform particle size, spherical shape and small relative velocities between the two phases, then the net effect of the particles on the fluid through a porous medium is equivalent to an extra body force term per unit volume $\frac{K N}{\epsilon}\left(\boldsymbol{q}_{d}-\boldsymbol{q}\right)$. Since the force exerted by the fluid on the particles is equal and opposite to that exerted by the particles on the fluid, there must be an extra force term, equal in magnitude but opposite in sign, in the equations of motion of the particles. The distances between particles are assumed to be so large compared with their diameter that inter-particle 
reactions need not be accounted for. The effects of pressure, gravity and Darcian force on the suspended particles, assumed large distances apart, are negligibly small and therefore ignored. If $m N$ is the mass of particles per unit volume, then the equation of motion and continuity for the particles, under the above assumptions, are

$$
\begin{aligned}
& m N\left[\frac{\partial \boldsymbol{q}_{d}}{\partial t}+\frac{1}{\epsilon}\left(\boldsymbol{q}_{d} \cdot \nabla\right) \boldsymbol{q}_{d}\right]=K N\left(\boldsymbol{q}-\boldsymbol{q}_{d}\right), \\
& \in \frac{\partial N}{\partial t}+\nabla \cdot\left(N \boldsymbol{q}_{d}\right)=0 .
\end{aligned}
$$

Since the density of a fluid particle moving with the fluid remains unchanged, we have

$$
\in \frac{\partial \rho}{\partial t}+(\boldsymbol{q} \cdot \nabla) \rho=0
$$

Let $\delta \rho, \delta p, \boldsymbol{q}(u, v, w)$ and $\boldsymbol{q}_{d}(l, r, s)$ denote respectively the perturbations in density $\rho$, pressure $p$, fluid velocity $(0,0,0)$ and particle velocity $(0,0,0)$. Then the linearized perturbation equations of the RivlinEricksen fluid-particle layer are

$$
\begin{aligned}
& \frac{\rho}{\in} \frac{\partial \boldsymbol{q}}{\partial t}=-\nabla \delta p-g \delta \rho \lambda+\frac{K N}{\epsilon}\left(\boldsymbol{q}_{d}-\boldsymbol{q}\right)-\frac{1}{k_{l}}\left(\mu+\mu^{\prime} \frac{\partial}{\partial t}\right) \boldsymbol{q}, \\
& \nabla \cdot \boldsymbol{q}=0 \\
& \left(\frac{m}{K} \frac{\partial}{\partial t}+1\right) \boldsymbol{q}_{d}=\boldsymbol{q}, \\
& \in \frac{\partial}{\partial t} \delta \rho=-w(D \rho), \\
& \frac{\partial M}{\partial t}+\nabla \cdot \boldsymbol{q}_{d}=0
\end{aligned}
$$

where $M=\frac{\in N}{N_{0}}$ and $N_{0}, N$ stand for the initial uniform number density and perturbation in number density respectively and $D=\frac{d}{d z}$.

Analyzing the disturbances into normal modes, we seek solutions whose dependence on $x, y$ and $t$ is given by

$$
\exp .\left(i k_{x} x+i k_{y} y+n t\right)
$$


where $k_{x}, k_{y}$ are the wave numbers along the $x$ - and $y$-directions, respectively, $k\left[=\left(k_{x}^{2}+k_{y}^{2}\right)^{\frac{1}{2}}\right]$, is the resultant wave number, $n$ is the growth rate which is, in general, a complex constant.

For perturbations of the form (2.11); Eqs (2.6)- (2.9), after elimination $\boldsymbol{q}_{d}$, yield

$$
\begin{aligned}
& \frac{\rho}{\epsilon} n u=-i k_{x} \delta p-\frac{1}{k_{l}}\left(\mu+\mu^{\prime} n\right) u-\frac{m N}{\epsilon(\tau n+1)} n u, \\
& \frac{\rho}{\epsilon} n v=-i k_{y} \delta p-\frac{1}{k_{l}}\left(\mu+\mu^{\prime} n\right) v-\frac{m N}{\epsilon(\tau n+1)} n v, \\
& \frac{\rho}{\epsilon} n w=-D \delta p-\frac{1}{k_{l}}\left(\mu+\mu^{\prime} n\right) w-\frac{m N}{\in(\tau n+1)} n w-g \delta \rho, \\
& i k_{x} u+i k_{y} v+D w=0, \\
& \in n \delta \rho=-(D \rho) w
\end{aligned}
$$

where $\tau=\frac{m}{K}$. Eliminating $\delta p$ between Eqs (2.12)-(2.14) and using Eqs (2.15) and (2.16), we obtain

$$
\begin{aligned}
& n\left[D\left(\frac{\rho}{\epsilon} D w\right)-\frac{k^{2}}{\epsilon} \rho w\right]+\left[D\left\{\left(\frac{\mu}{k_{l}}+\frac{\mu^{\prime}}{k_{l}} n\right) D w\right\}-k^{2}\left(\frac{\mu}{k_{l}}+\frac{\mu^{\prime}}{k_{l}} n\right) w\right]+ \\
& +\frac{n}{\in(\tau n+1)}\left\{D[m N D w]-k^{2} m N w\right\}=-\frac{g k^{2}}{\in n}(D \rho) w .
\end{aligned}
$$

\section{Two uniform fluids separated by a horizontal boundary}

Consider the case of two uniform Rivlin-Ericksen viscoelastic fluids of densities, viscosities, viscoelasticities, suspended particles number densities as $\rho_{2}, \mu_{2}, \mu_{2}^{\prime}, N_{2}$ and $\rho_{1}, \mu_{1}, \mu_{l}^{\prime}, N_{1}$ separated by a horizontal boundary at $z=0$. The subscripts 1 and 2 distinguish the lower and the upper fluids respectively. The medium porosity $\in$ is assumed to be the same in both the regions. Let the medium permeabilities of upper $(z>0)$ and lower $(z<0)$ media be $k_{12}$ and $\mathrm{k}_{11}$, respectively.

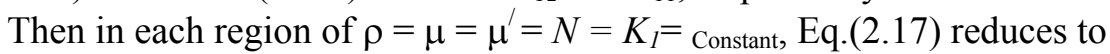

$$
\left(D^{2}-k^{2}\right) w=0 .
$$

The general solution of Eq.(3.1) is

$$
w=A e^{+k z}+B e^{-k z}
$$

where $A$ and $B$ are arbitrary constants. Then the boundary conditions to be satisfied in the present problem are

(i) The velocity $w \rightarrow 0$ when $z \rightarrow \infty$ (for the upper fluid) and $z \rightarrow-\infty$ (for the lower fluid).

(ii) $\quad w(z)$ is continuous at $z=0$. 
(iii) The jump condition at the interface $z=0$ between the fluids is obtained by integrating Eq.(2.17) across the interface $z=0$ and is

$$
\begin{aligned}
& \frac{n}{\epsilon}\left(\rho_{2} D w_{2}-\rho_{1} D w_{1}\right)_{z=0}+\left[\left(\frac{\mu_{2}}{k_{12}}+\frac{\mu_{2}^{\prime}}{k_{12}} n\right) D w_{2}-\left(\frac{\mu_{1}}{k_{11}}+\frac{\mu_{1}^{\prime}}{k_{11}} n\right) D w_{1}\right]_{z=0}+ \\
& +\frac{m n}{\in(\tau n+1)}\left[N_{2} D w_{2}-N_{1} D w_{1}\right]_{z=0}=-g \frac{k^{2}}{\in n}\left(\rho_{2}-\rho_{1}\right) w_{0}
\end{aligned}
$$

where $w_{0}$ is the common value of $w$ at $z=0$.

Applying boundary conditions (i) and (ii), we can write

$$
\begin{aligned}
& w_{1}=A e^{k z}, \quad(z<0), \\
& w_{2}=A e^{-k z}, \quad(z>0)
\end{aligned}
$$

where the same constant $A$ has been considered to ensure the continuity of $\mathrm{w}$ at $z=0$. Applying condition (3.3) to solutions (3.4) and (3.5), we obtain

$$
\begin{aligned}
& {\left[1+\left(\frac{\alpha_{2} \mathrm{v}_{2}^{\prime} \in}{k_{12}}+\frac{\alpha_{1} \mathrm{v}_{1}^{\prime} \in}{k_{11}}\right)\right] n^{3}+\left[1+m\left(\frac{N_{2}+N_{1}}{\rho_{2}+\rho_{1}}\right)+\tau\left(\frac{\alpha_{2} \mathrm{v}_{2} \in}{k_{12}}+\frac{\alpha_{1} \mathrm{v}_{1} \in}{k_{11}}\right)+\right.} \\
& \left.+\left(\frac{\alpha_{2} \mathrm{v}_{2}^{\prime} \in}{k_{12}}+\frac{\alpha_{1} \mathrm{v}_{1}^{\prime} \in}{k_{11}}\right)\right] n^{2}+\left[\left(\frac{\alpha_{2} \mathrm{v}_{2} \in}{k_{12}}+\frac{\alpha_{1} \mathrm{v}_{1} \in}{k_{11}}\right)-g k \tau\left(\alpha_{2}-\alpha_{1}\right)\right] n-g k\left(\alpha_{2}-\alpha_{1}\right)=0
\end{aligned}
$$

where

$$
\mathrm{v}_{1,2}=\frac{\mu_{1,2}}{\rho_{1,2}}, \quad \mathrm{v}_{1,2}^{\prime}=\frac{\mu_{1,2}^{\prime}}{\rho_{1,2}^{\prime}}, \quad \text { and } \quad \alpha_{1,2}=\frac{\rho_{1,2}}{\rho_{1}+\rho_{2}} \text {. }
$$

So $\alpha_{1}+\alpha_{2}=1$.

\section{Discussion}

\section{(a) Stable case $\left(\alpha_{2}<\alpha_{1}\right)$}

For the potentially stable arrangement $\left(\alpha_{2}<\alpha_{1}\right)$, there is no change in the coefficients of Eq.(3.6). Therefore, all the three roots of Eq.(3.6) are either real, negative or there is one real, negative root and the other two complex conjugates with negative real parts. The system is, therefore, stable in each case.

We thus conclude that the superposed and potentially stable arrangement of two Rivlin-Ericksen elastico-viscous fluids of different permeabilities in the presence of suspended particles in a porous medium is found to be really stable. This is in an agreement with the stability of Newtonian superposed fluids permeated with suspended particles in a porous medium, where also the system is always stable for the stable arrangement.

However, this is in contrast to the stability of Walters'(model B') superposed fluids permeated with suspended particles in a porous medium, with variable permeability and for the potentially stable where the system can be stable or unstable as the kinematic viscoelasticity is less than or greater than the medium permeability divided by medium porosity, in each region. 


\section{(b) Unstable case $\left(\alpha_{2}>\alpha_{1}\right)$}

For the potentially unstable arrangement $\left(\alpha_{2}>\alpha_{1}\right)$, the constant term in Eq.(3.6) is negative. Equation (3.6) has a change of sign and hence allows one positive root. The occurrence of a positive root implies instability of the system.

We, therefore, conclude that for two superposed Rivlin Ericksen elastico-viscous fluids permeated with suspended particles in a porous medium with different permeability and for the potentially unstable arrangement, the system is unstable. This is in agreement with the stability of Newtonian superposed fluids permeated with suspended particles in a porous medium with variable permeability, where also the system is always unstable for the unstable arrangement.

\section{Effect of a variable horizontal magnetic field}

Here we consider a static state in which an incompressible, infinitely electrically conducting RivlinEricksen elastico-viscous fluid is arranged in horizontal strata in a porous medium with variable permeability in the presence of suspended particles and a variable horizontal magnetic field $\boldsymbol{H}(H(z), 0,0)$. Let $\boldsymbol{h}\left(h_{x}, h_{y}, h_{z}\right)$ denote the perturbation in the magnetic field and $\mu_{e}$ stands for the magnetic permeability. Then the linearized hydromagnetic perturbation equations, relevant to the problem, are

$$
\begin{aligned}
& \frac{\rho}{\in} \frac{\partial \boldsymbol{q}}{\partial t}=-\nabla \delta p-g \delta \rho \lambda+\frac{k N}{\epsilon}\left(\boldsymbol{q}_{d}-\boldsymbol{q}\right)-\frac{1}{k_{l}}\left(\mu+\mu^{\prime} \frac{\partial}{\partial t}\right) \boldsymbol{q}+ \\
& +\frac{\mu_{e}}{4 \pi}[(\nabla \times \boldsymbol{h}) \times \boldsymbol{H}+(\nabla \times \boldsymbol{H}) \times \boldsymbol{h}], \\
& \nabla \cdot \boldsymbol{h}=0 \\
& \in \frac{\partial \boldsymbol{h}}{\partial t}=\nabla \times(\boldsymbol{q} \times \boldsymbol{H}),
\end{aligned}
$$

together with Eqs (2.7)- (2.9). For perturbation of the form Eq.(2.11), by writing component equations and eliminating $u, v, h_{x}, h_{y}, h_{z}, \delta \rho$ and $\delta p$ amongst Eqs (5.1)-(5.3) and (2.7)- (2.9), we obtain

$$
\begin{aligned}
& n\left[D\left(\frac{\rho}{\epsilon} D w\right)-\frac{k^{2}}{\epsilon} \rho w\right]+\left\{\left[D\left(\frac{\mu}{k_{l}}+\frac{\mu^{\prime}}{k_{l}} n\right) D w\right]-k^{2}\left(\frac{\mu}{k_{l}}+\frac{\mu^{\prime}}{k_{l}} n\right)\right\}+ \\
& +\frac{n}{\in(\tau n+1)}\left[D(m N D w)-k^{2} m N w\right]+\frac{\mu_{e} k_{x}^{2}}{4 \pi n \in}\left[D\left(H^{2} D w\right)-H^{2} k^{2} w\right]=-\frac{g k^{2}}{\in n}(D \rho) w .
\end{aligned}
$$

\section{Two uniform visco-elastic fluids separated by a horizontal boundary}

Here we consider the case of two uniform, Rivlin- Ericksen elastico-viscous densities, viscosities, viscoelasticities, suspended particles number densities, magnetic fields as $\rho_{2}, \mu_{2}, \mu_{2}^{\prime}, N_{2}, H_{2}$ and $\rho_{l}, \mu_{l}, \mu_{l}^{\prime}$, $N_{l}, H_{l}$ separated by a horizontal boundary $z=0$ in a porous medium. Let the medium permeabilities of upper $(z>0)$ and lower $(z<0)$ media be $k_{12}$ and $k_{11}$, respectively. 


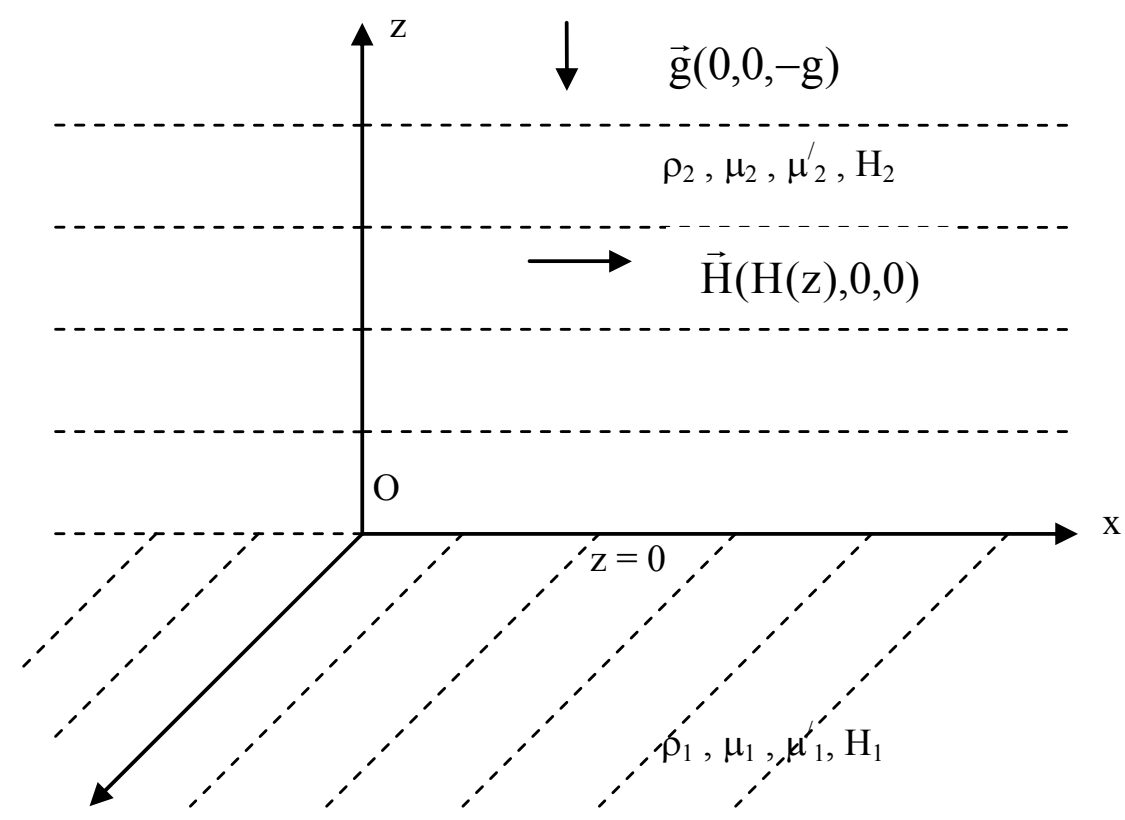

Fig.1. Two uniform Rivlin-Ericksen elastico-viscous fluids separated by a horizontal boundary $z=0$. reduces to

Then in each region of constant $\rho$, constant $\mu$, constant $\mu^{\prime}$, constant $m N$, constant $k_{l}$, Eq.(5.4)

$$
\left(D^{2}-k^{2}\right) w=0
$$

The boundary conditions to be satisfied in the present problem are (i) the vanishing of w at $z \rightarrow+\infty$ and $z \rightarrow-\infty$, (ii) continuity of $\mathrm{w}$ at $z=0$ and (iii) the jump condition. The jump condition is obtained by integrating Eq.(5.4) across the interface at $z=0$ and is

$$
\begin{aligned}
& \frac{n}{\in}\left(\rho_{2} D w_{2}-\rho_{1} D w_{1}\right)_{z=0}+\left[\left(\frac{\mu_{2}}{k_{12}}+\frac{\mu_{2}^{\prime}}{k_{12}} n\right) D w_{2}-\left(\frac{\mu_{2}}{k_{11}}+\frac{\mu_{2}^{\prime}}{k_{11}} n\right) D w_{1}\right]_{z=0}+\frac{m n}{\in(\tau n+1)}+ \\
& +\left[N_{2} D w_{2}-N_{1} D w_{1}\right]_{z=0}+\frac{\mu_{e} k_{x}^{2}}{4 \pi n \in}\left(H_{2}^{2} D w_{2}-H_{1}^{2} D w_{1}\right)_{z=0}=-g \frac{k^{2}}{\in n}\left(\rho_{2}-\rho_{1}\right) w_{0},
\end{aligned}
$$

Applying boundary conditions (i) and (ii), the solution of Eq.(6.1) is given by Eqs (3.4) and (3.5). Applying the condition (6.2) to solutions (3.4) and (3.5), we obtain

$$
\begin{aligned}
& {\left[1+\left(\frac{\alpha_{2} \mathrm{v}_{2}^{\prime} \in}{k_{12}}+\frac{\alpha_{1} \mathrm{v}_{1}^{\prime} \in}{k_{11}}\right)\right] n^{3}+\left[1+m\left(\frac{N_{2}+N_{1}}{\rho_{2}+\rho_{1}}\right)+\tau\left(\frac{\alpha_{2} \mathrm{v}_{2} \in}{k_{12}}+\frac{\alpha_{1} \mathrm{v}_{1} \in}{k_{11}}\right)+\left(\frac{\alpha_{2} \mathrm{v}_{2}^{\prime} \in}{k_{12}}+\frac{\alpha_{1} \mathrm{v}_{1}^{\prime} \in}{k_{11}}\right)\right] n^{2}+} \\
& +\left[\left(\frac{\alpha_{2} \mathrm{v}_{2} \in}{k_{12}}+\frac{\alpha_{1} \mathrm{v}_{1} \in}{k_{11}}\right)+\tau k_{x}^{2}\left(V_{A 2}^{2}+V_{A 1}^{2}\right)-g k \tau\left(\alpha_{2}-\alpha_{1}\right)\right] n+\left[k_{x}^{2}\left(V_{A 2}^{2}+V_{A 1}^{2}\right)\right]-g k\left(\alpha_{2}-\alpha_{1}\right)=0
\end{aligned}
$$


where $v_{1,2}=\frac{\mu_{1,2}}{\rho_{1,2}}, v_{1,2}^{\prime}=\frac{\mu_{1,2}^{\prime}}{\rho_{1,2}^{\prime}}$, and $\alpha_{1,2}=\frac{\rho_{1,2}}{\rho_{1}+\rho_{2}}, V_{A 2}^{2}=\frac{\mu_{e} H_{2}^{2}}{4 \pi\left(\rho_{1}+\rho_{2}\right)}$ and $V_{A 1}^{2}=\frac{\mu_{e} H_{1}^{2}}{4 \pi\left(\rho_{2}+\rho_{1}\right)}$.

\section{Discussion}

\section{(a) Stable case $\left(\alpha_{2}<\alpha_{1}\right)$}

For the potentially stable arrangement $\left(\alpha_{2}<\alpha_{1}\right)$, there is no change of sign in the coefficients of Eq.(6.3). The system is, therefore, stable. Therefore, we conclude that for two superposed RivlinEricksen elastico-viscous fluids permeated with suspended particles in a porous medium with variable permeability in hydromagnetics and for the potentially stable arrangement, the system is always stable. The result is the same in the hydrodynamic case. However, this is in contrast to the stability of Walters'(model $\mathrm{B}^{\prime}$ ) superposed fluids permeated with suspended particles in a porous medium, with variable permeability and for the potentially stable case where the system can be stable or unstable depending on the values of the kinematic viscoelasticity and the medium permeability divided by the medium porosity, in each region.

\section{(b) Unstable case $\left(\alpha_{2}>\alpha_{1}\right)$}

For the potentially unstable arrangement $\left(\alpha_{2}>\alpha_{1}\right)$, if $k_{x}^{2}\left(V_{A 1}^{2}+V_{A 2}^{2}\right)>g k\left(\alpha_{2}-\alpha_{1}\right)$. Equation (6.3) does not admit any change of sign and so has no positive root. Therefore the system is stable. However, if $k_{x}^{2}\left(V_{A 1}^{2}+V_{A 2}^{2}\right)<g k\left(\alpha_{2}-\alpha_{1}\right)$, the constant term in Eq.(6.3) is negative, therefore allows one change in sign, which means it has one positive root. The occurrence of a positive root implies that the system is unstable.

Thus, for the potentially unstable case, the magnetic field has got a stabilizing effect and completely stabilizes the system for all wave numbers which satisfy the inequality $k_{x}^{2}\left(V_{A 1}^{2}+V_{A 2}^{2}\right)>g k\left(\alpha_{2^{-}}\right.$ $\alpha_{1}$ ), that is $k>k^{*}$. Where $k^{*}=\frac{g\left(\alpha_{2}-\alpha_{1}\right)}{V_{A_{2}}^{2}+V_{A_{1}}^{2}} \sec ^{2} \theta$, here $\theta$ is the angle between $k_{x}$ and $k$ such that $k_{x}=k$ $\cos \theta$. However, for the potentially unstable arrangement, the system is unstable for the wave number band $k<k^{*}$.

We therefore conclude that for two superposed Rivlin-Ericksen elastico-viscous fluids permeated with suspended particles in a porous medium (with different permeability) and for the potentially unstable arrangement, the magnetic field succeeds in stabilizing certain wave-number bands which were unstable in the absence of the magnetic field. The system can thus be stable or unstable. This is in agreement with the stability of Newtonian superposed fluids permeated with suspended particles in a porous medium.

\section{Conclusion}

Hydrodynamic and hydromagnetic stability of the superposed Rivlin-Ericksen elastico-viscous fluid of different permeability with a porous medium has been investigated. The principle concluding remarks in both cases separately are as below. 


\section{Effect of suspended particles}

(i): In case of potentially stable arrangement of the Rivlin-Ericksen fluids in the presence of a porous medium the system is found to be stable, which is an agreement with the stability of Newtonian superposed fluid, where the system is always stable.

(ii): However, this is in contrast to the stability of Walters'(Model B') superposed fluids permeated with suspended particles in a porous medium, with variable permeability and for the potentially stable case where the system can be stable or unstable depending on the kinematic viscoelasticity and the medium permeability divided by the medium porosity, in each region.

(iii): In case of the potentially unstable arrangement, the system is unstable, for a superposed fluid of different permeability.

(iv): The magnetic field succeeds in stabilizing certain wave-number bands which were unstable in the absence of the magnetic field. The system can thus be stable or unstable. This is in agreement with the stability of Newtonian superposed fluids permeated with suspended particles in a porous medium in hydromagnetics.

\section{Effect of variable horizontal magnetic field}

(i): In case of the potentially stable arrangement, the system is always stable, similarly as in the hydrodynamic case.

(ii): However, this is in contrast to the stability of Walters'(Model B') superposed fluids permeated with suspended particles in a porous medium, with variable permeability and for the potentially stable case where the system can be stable or unstable depending of the kinematic viscoelasticity and the medium permeability divided by medium porosity, in each region.

(iii): For the potentially unstable case, the magnetic field has got a stabilizing effect for all wave numbers with $k>k^{*}$ and unstable for $k<k^{*}$.

(iv): Graphic representation of two superposed fluids has been studied here.

\section{Nomenclature}

$$
\begin{aligned}
g & - \text { acceleration due to gravity }\left(\mathrm{ms}^{-2}\right) \\
\boldsymbol{H} & - \text { magnetic field intensity vector having component }(\mathrm{H}(\mathrm{Z}), 0,0) \\
K & - \text { Stoke's drag coefficient }\left(\mathrm{kgs}^{-1}\right) \\
k & - \text { wave number }\left(\mathrm{m}^{-1}\right) \\
k_{x}, k_{y} & - \text { horizontal wave numbers }\left(\mathrm{m}^{-1}\right) \\
m & - \text { mass of single particle }(\mathrm{g}) \\
N & - \text { suspended particle number density }\left(\mathrm{m}^{-3}\right) \\
p & - \text { fluid pressure }(\mathrm{Pa}) \\
v & - \text { suspended particle velocity }\left(\mathrm{ms}^{-1}\right) \\
\in & - \text { medium porosity }\left(\mathrm{m}^{0} \mathrm{~s}^{0} k^{0}\right) \\
\eta & - \text { electrical resistivity }(-) \\
\mu & - \text { dynamic viscosity }\left(\mathrm{km}^{-1} \mathrm{~s}^{-1}\right) \\
\mu^{\prime} & - \text { fluid viscoelasticity }\left(\mathrm{km}^{-1} \mathrm{~s}^{-1}\right)
\end{aligned}
$$


$\mu_{e}$ - magnetic permeability

$\rho-\operatorname{density}\left(\mathrm{kgm}^{-3}\right)$

$v-$ kinematic viscosity $\left(m^{2} s^{-1}\right)$

$v^{\prime}-$ kinematic viscoelasticity $\left(m^{2} s^{-1}\right)$

\section{Acknowledgement}

The authors are grateful to the referees for their technical comments and valuable suggestions, resulting in a significant improvement of the paper.

\section{References}

Bhatia P.K. (1974): Rayleigh-Taylor instability of two viscous superposed conducting fluids. - Nuovo Cimento, vol.19B, pp.161-169.

Chandra K. (1938): Instability of fluid heated from below. - Proc. Roy. Soc. (Lon.), vol.164A, pp.231-242.

Gupta U., Aggarwal P. and Wanchoo R.K. (2012): Thermal convection of dusty compressible Rivlin-Ericksen viscoelastic fluid with Hall currents. - Thermal Science, vol.16, No.1, pp.177-191.

Kent A. (1966): Instability of laminar flow of a magneto-fluid. - Phys. Fluids, vol.9, No.7, pp.1286-1289.

Kumar P., Lal R. and Singh M. (2007): Hydrodynamic and hydromagnetic stability of two stratified Rivlin-Ericksen elastico-viscous superposed. - Int. J. of Applied Mechanics and Engineering, vol.12, No.3, pp.645-653.

Rivlin R.S. and Ericksen J.L. (1955): Stress-deformation relations for isotropic materials. - J. Rational Mech. and Analysis, vol.4, pp.323-425.

Scanlon J.W. and Segel L.A. (1973): Some effects of suspended particles on the onset of Bénard convection. - Phys. Fluids, vol.16, pp.1573-1578.

Sharma R.C. (1976): Effect of rotation on thermal instability of a viscoelastic fluid. - Acta Physica Hungarica, vol.40, pp.11-17.

Sharma R.C. and Kumar P. (1997): Hyderomagnetic stability of two Rivlin-Ericksen elastico-viscous superposed conducting fluids. - Z. Naturforsch, vol.52a, pp.528-532.

Sharma R.C. and Sharma K.C. (1977): Rayleigh-Taylor Instability of two superposed conducting fluids in the presence of suspended particles. - Acta Physica Hungarica, vol.43, pp.251-258.

Sharma R.C., Kirti Prakash and Dube S.N. (1976): Effect of suspended particles on the onset of Bénard convection in hydromagnetics. - Acta Physica Hungarica, vol.40, pp.3-10.

Singh M. and Gupta R.K. (2011): Thermal instability of Rivlin-Ericksen elastico-viscous fluid permeated with suspended particles in hydrodynamics in a porous medium. - Int. J. of Applied Mechanics and Engineering, vol.16, No.4, pp.1169-1179.

Srivastava L.P. (1971): Unsteady flow of Rivlin-Ericksen fluid with suspended particles. - Istanbul Teknik University, Bulletin, vol.194, pp.07-19.

Srivastava R.K. and Singh K.K. (1988): Drag on a sphere oscillating in a conducting dusty viscous fluid in presence of uniform magnetic field. - Bull. Cal. Math. Soc., vol.80, pp.286-292. 